\title{
Development of Dry Skin in the NOA Mouse Under Individual Housing Conditions: A Potentially Useful Animal Model for Evaluating Moisturizing Effects
}

\author{
Taizo KONDO'), Hitoshi ${ }^{1} \mathrm{HNO}^{2)}$, Toshio KONDO ${ }^{1)}$, \\ Yasuhisa SHIOMOTO1), and Akira MOMII')
}

\begin{abstract}
1)Division of Pharmacology, Drug Safety and Metabolism, and ${ }^{2)}$ Pharmaceutical Technology Institute, Otsuka Pharmaceutical Factory, Inc., 115 Tateiwa, Muya-cho, Naruto, Tokushima 772-8601, Japan
\end{abstract}

\begin{abstract}
In a previous study, we reported the development of grossly observable dry skin in all of the Naruto Research Institute Otsuka Atrichia (NOA) mice that were housed individually. In the present study, dermal physiological function tests were conducted and the usefulness of this dry skin model for evaluating the efficacy of topical moisturizers was assessed. As a result, we have confirmed a marked reduction in the water content of the stratum corneum in these animals. Therefore, the development of dry skin in the NOA mouse strain under individual housing conditions may be expected to serve as a useful animal model for evaluating topical moisturizers. Specifically, the water content of the stratum corneum was restored in proportion to the oil content of the ointment base used to treat the animals, and the moisturizing effects of urea were confirmed in animals treated with urea-containing ointment. In addition, when the animals that had been housed individually were returned to group housing conditions, the water content of the stratum corneum was restored, with a corresponding improvement in dry skin. This finding suggests that socio-psychological factors are involved in the etiology of dry skin in individually housed NOA mice.
\end{abstract}

Key words: animal model, dry skin, individual housing, moisturizing effects, NOA mouse

\section{Introduction}

The Naruto Research Institute Otsuka Atrichia (NOA) mouse is a hair-deficient mutant [13] that has been established as an inbred strain in our facility. This mouse strain spontaneously develops ulcerative and erosive wet skin lesions at a high rate [13]. In a previous study, we investigated the effects of housing conditions on the onset and prevalence of such wet skin lesions, and the results suggested the involvement of socio-psychological factors in the etiology of these lesions [14]. In addition, we noted new grossly observable characteristics with special regard to the development of dry skin in all of the NOA mice under individual housing conditions. Therefore, based on the observation that dry skin develops in individually housed NOA mice, this

(Received 11 March 2005 / Accepted 22 July 2005)

Address corresponding: T. Kondo, Division of Pharmacology, Drug Safety and Metabolism, Otsuka Pharmaceutical Factory, Inc., 115

Tateiwa, Muya-cho, Naruto, Tokushima 772-8601, Japan 
strain may be expected to serve as a useful animal model for evaluating the efficacy of topical moisturizers [14]. In the present study, dermal physiological function tests were carried out in order to more clearly characterize this dry skin model. In the dermal physiological function tests, the HR-1 mouse which is an established strain of hairless $(h / h)$ mouse was used for comparison. Based on the findings of these tests, we assessed the usefulness of this model for evaluating the efficacy of topical moisturizers. No suitable animal model for this purpose has been reported to date.

\section{Materials and Methods}

This study was carried out using SPF animals under a barrier system. All of the NOA mice used were bred at our facility. The breeding conditions were described in our previous report [14]. SAS software (SAS Institute Japan, Ltd., Tokyo, Japan) was used for statistical analysis.

Dermal physiological function tests of the dry skin that developed under individual housing conditions

Twenty 4-week-old male NOA mice and fourteen 4week-old male HR-1 mice (Hos:HR-1) were studied. The HR-1 mouse is an established strain of hairless mouse $(h / h)$, and the HR-1 mice used in this study were obtained from Hoshino Laboratory Animals (Saitama, Japan) for comparison. The mice were housed under non-littermate group housing conditions for 1 week at 5 mice/cage for the NOA mice and at 3 or 4 mice/cage for the HR-1 mice. With regard to the NOA mice, 10 animals were subsequently transferred to individual housing conditions and the other 10 animals were kept under group housing conditions. With regard to the HR-1 mice, 7 animals were transferred to individual housing conditions and the other 7 animals were kept under group housing conditions. All of the mice underwent dermal physiological function tests of the cervico-dorsal skin once a week for a period of 6 weeks.

The water content of the stratum corneum, sebum quantity, and $\mathrm{pH}$ value on the skin surface were measured using a CORNEOMETER $^{\circledR}$ CM825, a $^{\circledR}$ SEBUMETER $^{\circledR}$ SM810, and a SKIN-pH-METER ${ }^{\circledR}$ PH900 (CK Electronic GmbH, Köln, Germany), respectively. For each animal, determinations were carried out at about 3:00 P.M. for the water content of the stratum corneum, sebum quantity, and $\mathrm{pH}$ value on the skin surface, in that order. The mouse was held in the hand of the person making the measurements, and the probe was applied to the skin of the cervico-dorsal region to measure the water content of the stratum corneum and skin $\mathrm{pH}$. For measurement of sebum quantity, the skin of the cervico-dorsal region was pulled up by the person performing the measurement before the probe was applied in order to minimize possible compression effects resulting from direct application of the probe (pressure of $0.3 \mathrm{~N}$ applied for $30 \mathrm{~s}$ ).

The effects of housing conditions on dermal physiological function were analyzed by Student's $t$-test.

\section{Changes in the water content of the stratum corneum after changing housing conditions}

Twenty-four 9-week-old male NOA mice with dry skin that had developed under individual housing conditions from 5 weeks of age were prepared. The animals were returned to group housing conditions at 4 mice/ cage in 6 subgroups (A-F). To serve as controls, 10 additional animals were kept under individual housing conditions, and 10 additional animals were kept under group housing conditions at $5 \mathrm{mice} / \mathrm{cage}$. The water content of the stratum corneum in the skin of the cervico-dorsal region was measured once a week for a period of 6 weeks, and the results were analyzed by Student's $t$-test. Before each measurement, the nesting state in each cage was noted in order to serve as an index of the social environment in the cage.

\section{Assessment of the usefulness of this animal model for} the evaluation of moisturizing effects

A total of fifty 4-week-old male NOA mice were used. After the animals were reared under non-littermate group housing conditions at 5 mice/cage for 1 week, they were transferred to individual housing conditions for an additional 4 weeks. Ten animals were allocated to each application group and to a control group. The animals in the control group were not treated with ointment. After measurement of the water content of the stratum corneum in the skin of the cervico-dorsal region, a test article was applied to the entire area of the dorsal skin. Four types of ointment were used as test articles: two ointment bases containing $15 \%$ and $25 \%$ oil, and the same ointment bases with the addition of $10 \%$ urea. The ointments studied 

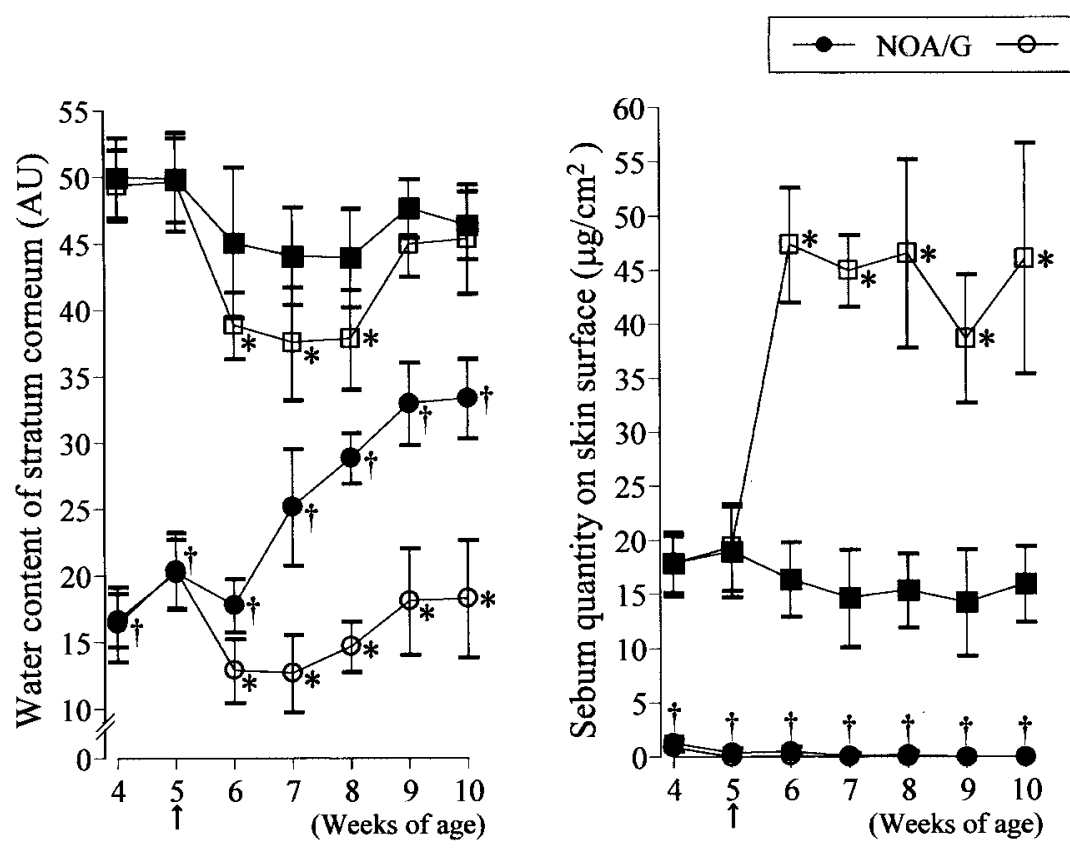
NOA/I $-\mathrm{HR}-1 / \mathrm{G} \rightarrow \mathrm{HR}-1 / \mathrm{I}$

Fig. 1. Changes in the values obtained by dermal physiological function tests of the cervico-dorsal skin under group housing and individual housing conditions, and strain differences. G: Group housing. I: Individual housing from 5 weeks of age (arrows). NOA: $\mathrm{n}=10$. HR-1: $\mathrm{n}=7$. AU: Arbitrary units. Vertical bars at each data point indicate mean \pm SD. *: Significant difference $(P<0.01)$ between individual housing and group housing conditions. $\dagger$ : Significant difference $(P<0.01)$ between NOA and HR-1 mice under group housing conditions.

were $\mathrm{O} / \mathrm{W}$-type ointments prepared using oils consisting of glycerin fatty acid ester, fatty alcohol, silicone oil, etc., according to our in-house recipe. Approximately $20 \mathrm{mg}$ of test article was applied to each animal. The water content of the stratum corneum was measured three times at 24-h intervals after application.

Tukey's test was used for statistical analysis of the moisturizing effects at $24 \mathrm{~h}$ after application. The effects were judged to be positive when the water content was significantly higher $(P<0.01)$ than that in the control group. The effects of urea on water content were also assessed.

The present study was approved by the Committee for the Care and Use of Laboratory Animals of Otsuka Pharmaceutical Factory, Inc.

\section{Results}

Dermal physiological function tests of the dry skin that developed under individual housing conditions

Figure 1 shows the changes in the water content of the stratum corneum, sebum quantity, and $\mathrm{pH}$ value on the skin surface of the cervico-dorsal region for each mouse strain under the two housing conditions. In comparison with the HR-1 mice, the NOA mice showed characteristic differences in skin physiology when housed under group housing conditions. Specifically, the water content of the stratum corneum and the sebum quantity were significantly lower $(P<0.01)$ and the $\mathrm{pH}$ value was significantly higher $(P<0.01)$ in the NOA mice as compared with the HR-1 mice. When the animals were transferred from group housing conditions to individual housing conditions, the NOA mice showed a marked decrease in water content in comparison with the animals that were kept under group housing conditions. This decrease in water content paralleled the development of dry skin as assessed by gross examination. The HR-1 mice that were housed individually also showed a transient decrease in water content, but within 5 weeks, the water content returned to the same level as in the animals kept under group housing conditions. In the HR-1 mice kept under individual housing conditions, the sebum quantity increased markedly and no dry skin was noted by gross examination, in contrast to the NOA mice. 
Changes in the water content of the stratum corneum after changing housing conditions

Figure 2 shows the changes in the water content of the stratum corneum in the skin of the cervico-dorsal region and the nesting state of the NOA mice when they were transferred from individual housing to group housing conditions. Restoration of the water content was observed in all of the subgroups, with a return to the levels seen in the animals kept under group housing conditions. However, the rate of restoration varied among the subgroups. In subgroups $\mathrm{B}, \mathrm{E}$, and $\mathrm{F}$, the animals formed a single nest $2-3$ weeks after they were transferred to group housing conditions, and this situation remained stable thereafter. A smooth restoration in the water content of the stratum corneum was seen, with no significant difference observed after 3 weeks as compared with the animals kept under group housing conditions. On the other hand, in subgroups $\mathrm{A}, \mathrm{C}$, and D, the total number of nests varied during the observation period. Restoration of the water content of the stratum corneum was slow. In subgroups $\mathrm{A}$ and $\mathrm{D}$, it barely reached the nonsignificant difference level after 7 weeks (the end of the observation period) as compared with the animals kept under group housing conditions. In subgroup $\mathrm{C}$, the animals followed an intermediate course. When the mice were transferred from individual housing to group housing conditions, gross examination showed a decrease in or the disappearance of skin exfoliation and the disappearance of dry skin, paralleling restoration of the water content of the stratum corneum.

Assessment of the usefulness of this animal model for the evaluation of moisturizing effects

Figure 3 shows the changes in the water content of the stratum corneum in the skin of the cervico-dorsal region after the application of ointment. The water content at $24 \mathrm{~h}$ after application in each application group was compared with the value for untreated animals that were kept under individual housing conditions. No statistically significant moisturizing effects were observed in the animals that were treated with the $15 \%$ oil base (15B), with no statistically significant difference in water content as compared with untreated animals that were kept under individual housing conditions (I). The water content increased significantly $(P<0.01)$ in the animals treated with the $25 \%$ oil ointment base or with either of the urea-containing ointments; these three ointments $(25 \mathrm{~B}, 15 \mathrm{U}, 25 \mathrm{U})$ were therefore judged to have moisturizing effects. Each urea-containing ointment (15U, 25U) was found to provide a further enhancement in water content as compared with the corresponding ointment base (15B or 25B).

\section{Discussion}

In the individually housed NOA mice, the water content of the stratum corneum in the skin of the cervico-dorsal region decreased markedly in parallel with the abnormal findings noted by gross observation. When NOA mice that had developed dry skin under individual housing conditions were returned to group housing conditions, the water content of the stratum corneum was restored, accompanied by the disappearance of skin exfoliation and dry skin. With regard to socio-psychological factors, it was found that each mouse initially made its own nest after being returned to group housing conditions, but the total number of nests then gradually decreased. This finding suggests that the stability of the social environment is inversely related to the total number of nests in a cage. Evaluation of the relationships between water content and the socio-psychological factors in each subgroup also suggests an association between the restoration of water content and an increase in social stability. In addition, these findings suggest that the water content of the stratum corneum varies according to changes in social stability and that the number of nests in a cage may be a useful index of the socio-psychological state of the animals. In brief, these findings suggest that various socio-psychological factors may be involved in the development of dry skin. In order to support this hypothesis, it would be desirable to conduct further investigations on the effects of social rank on water content, since a social ranking system has been described in mice kept under group housing conditions [5].

In dermal physiological function tests, if a haired mouse is used in the study, it is clear that hair clipping influences the test results. Given that the nude $(n u / n u)$ mutant mouse is athymic, the effects of differences in immunological status must be taken into consideration. Hairless $(h / h)$ mutant mice were therefore used for comparison. The HR-1 mouse is an established strain of hairless mutant mouse. Since a decrease in the water content of the stratum corneum was also observed in 

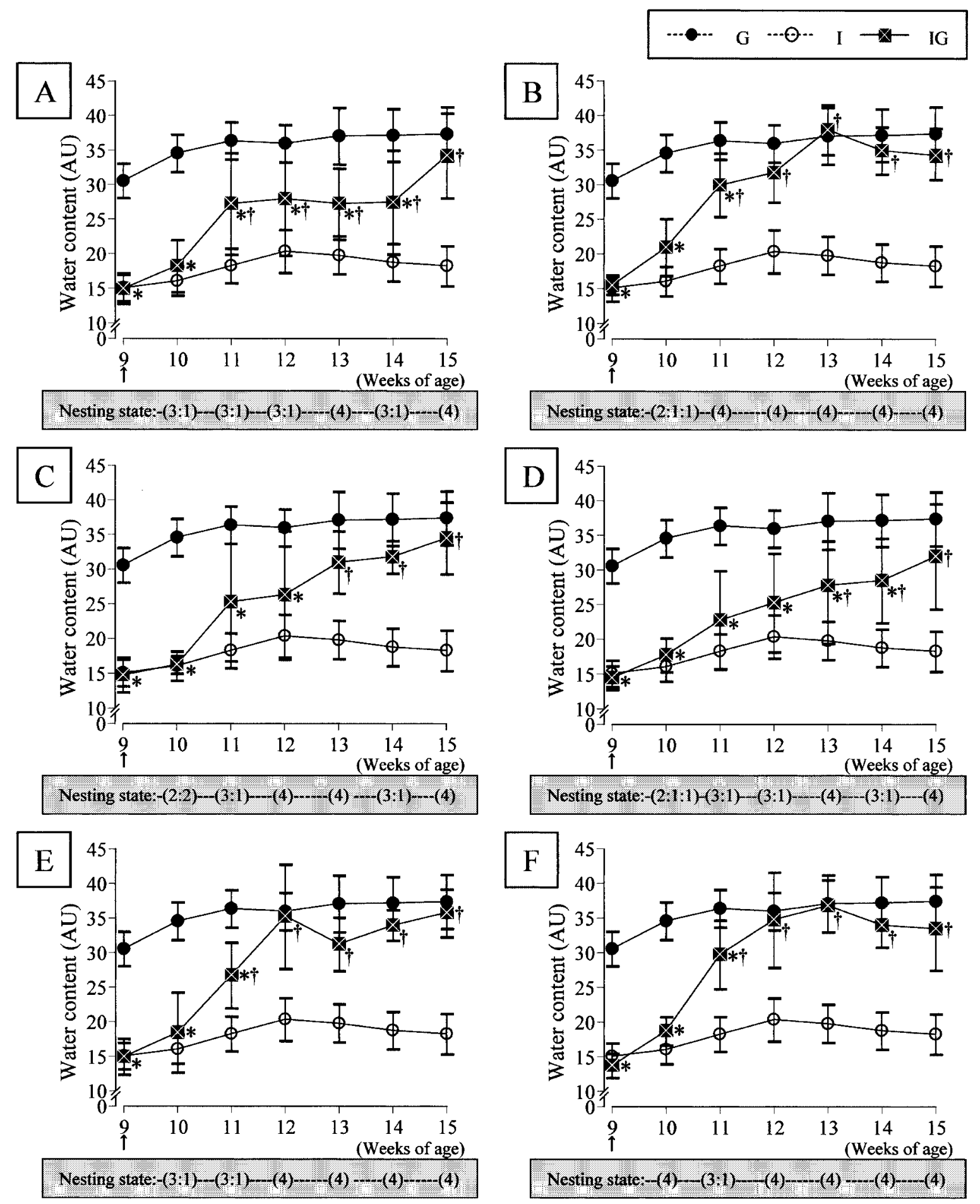

Fig. 2. Changes in the water content of the stratum corneum of the cervico-dorsal skin and nesting state after changing housing conditions. A-F: Subgroups (see text for details). G: Group housing (n=10). I: Individual housing from 5 weeks of age $(n=10)$. IG: Individual housing from 5 weeks of age, returned to group housing after 4 weeks (arrows, $\mathrm{n}=4$ for each subgroup). AU: Arbitrary units. Vertical bars at each data point indicate mean \pm SD. *: Significant difference $(P<0.01)$ vs. group housing. $\dagger$ : Significant difference $(P<0.01)$ vs. individual housing. Explanation of the nesting state: (2:1:1) 3 nests, 2 mice made 1 nest together and the remaining 2 mice made 1 nest each; (3:1) 2 nests, 3 mice made 1 nest together and the remaining 1 mouse made 1 nest; (2:2) 2 nests, 2 mice made 1 nest together, and the remaining 2 mice made 1 nest together; (4) 1 nest, 4 mice made 1 nest together. 


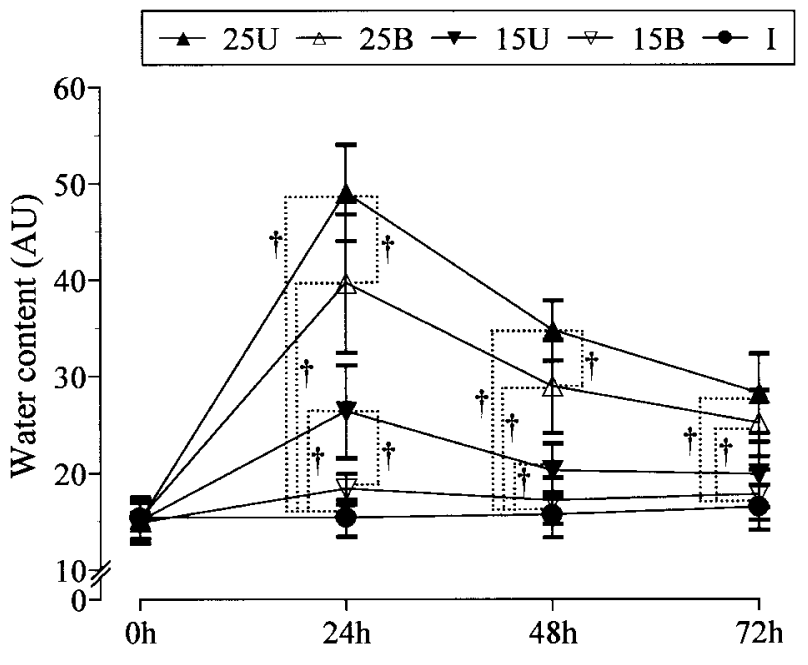

Fig. 3. Changes in the water content of the stratum corneum of the cervico-dorsal skin after the application of ointment. 25U: Urea-containing 25\% oil ointment. 25B: $25 \%$ oil ointment base. 15U: Urea-containing $15 \%$ oil ointment. 15B: $15 \%$ oil ointment base. I: Individual housing. $0 \mathrm{~h}: 9$ weeks of age (after 4 weeks of individual housing starting from 5 weeks of age). AU: Arbitrary units. $n=10$ in each group. Vertical bars at each data point indicate mean \pm SD. $\dagger: \mathrm{p}<0.01$.

HR-1 mice under individual housing conditions, the existence of a mechanism by which the water content of the skin is decreased by the stress of individual rearing in mice of this strain can be inferred. Aioi et al. [1] reported that placing $\mathrm{BALB} / \mathrm{c}$ mice under the stress of overcrowded housing (40 mice/cage) for 1 week was associated with exfoliation and wrinkle formation of the epidermis, a decrease in skin surface conductance, and decreases in ceramide and pyrrolidone carboxylic acid, which are moisturizing factors in the stratum corneum. Their report also suggested the existence of a mechanism in mice that decreases the water content of the skin in response to stress.

With regard to the HR-1 mice kept under individual housing conditions, no animals were found to have dry skin by gross examination, and after 5 weeks, the water content of the stratum corneum was restored to the level seen in animals kept under group housing conditions. At that time, a marked increase in the sebum quantity on the skin surface was observed, and this increase was thought to be a compensatory reaction to water loss from the skin. Therefore, although detailed examination is required, a compensatory mechanism that accelerates se- bum supply from the sebaceous glands in response to water loss from the skin appears to be present in mice. Ishida et al. [11] reported a marked decrease in the skin surface lipids of female hairless mice under overcrowded housing conditions (20 mice/cage). Tsuchiya and Horii [25] reported that lipogenesis in the sebaceous glands of the auricle of Syrian hamsters decreased under immobilization-induced stress conditions and that testosterone was involved in this decrease. Although the findings of these reports are exactly the opposite of the results of the present study in HR-1 mice, it is evident that stress can affect the sebaceous glands. Therefore, comparative evaluation of the effects of stress on the function of the sebaceous glands with respect to differences in the type and degree of stress, animal species, strain, sex, and site is required in order to clarify the relationships between dermal physiological function and stress.

The hairless state of the NOA mouse becomes conspicuous with growth. It has been confirmed histologically that cysts form in the hair follicles, and almost no hair ducts are found in the hairless state [13]. The results for the sebum quantity obtained in the present study agree with these histological findings, indicating that almost no sebum is produced by the sebaceous glands in the NOA mouse (Fig. 1). The skin characteristics of the NOA mouse are conspicuous hairlessness and a low water content of the stratum corneum, and these characteristics appear to contribute to the development of dry skin. This is supported by the measurements of the sebum quantity and water content of the stratum corneum (Fig. 1). In the HR-1 mouse, when the water content of the skin decreases, the supply of sebum from the sebaceous glands increases, and as a result, the water content is restored. In contrast, in the NOA mouse, the sebum quantity does not increase, the water content is not restored, and the water content of the skin, which is already low, decreases further, resulting in the development of dry skin.

It is clear that the $\mathrm{pH}$ value on the skin surface is altered by the housing conditions and strain differences. However, with regard to the influencing factors, further investigation is required.

Micro-environmental factors such as humidity and temperature were presumed to have an effect on the development of dry skin in individually housed NOA mice, but in an experiment in which 2-15 mice were housed per cage and the water content of the stratum 
corneum was measured, no differences in water content attributable to the number of mice per cage were found (unpublished data). According to a report by Aioi et al. [1], who used BALB/c mice, no differences in temperature or humidity were observed between housing at 5 mice/cage and housing at 40 mice/cage. From these results, it can be inferred that there were no differences in the temperature or humidity in the cages due to differences in the number of mice housed per cage in the present study. Therefore, micro-environmental factors do not appear to play an important role in the development of dry skin.

The involvement of stress in atopic dermatitis (AD), a typical allergic dermatopathy in humans, has been recognized for many years $[3,4,15,17]$. Recently, detailed evaluation of the relationships between $\mathrm{AD}$ and the psychological state has also begun in Japan $[9,10]$, and the interrelationship of psychological states such as depression, tension, and anxiety with the low water content of the stratum corneum in AD patients has been demonstrated. Dry skin is a typical disease state of AD $[6,8,26]$, and Werner [27] has reported low water content of the stratum corneum in this condition. The involvement of stress in the onset and aggravation of psoriasis has also been reported [2, 19, 20], and low water content of the stratum corneum [21] and decreased water-holding capacity of the skin [23] have been demonstrated. Therefore, the NOA mouse can be considered to be a suitable animal model for analyzing the relationships between dermatopathy and psychological factors as well as for evaluating the effects of psychological states on skin morphology and function.

In the assessment of the usefulness of the NOA mouse as an animal model for evaluating moisturizing effects (Fig. 3), 25\% oil ointment base was judged to have a moisturizing effect, but $15 \%$ oil ointment base was not. These results indicate that the dry skin that develops in NOA mice under individual housing conditions can provide useful data concerning the oil content in ointments. It was also demonstrated that this might be a useful model for evaluating the moisturizing effect of urea, based on the comparison of the results for the two urea-containing ointments and the corresponding ointment bases. These results indicate that the dry skin that develops in the NOA mouse under individual housing conditions is a useful model for evaluating the moisturizing effects of topical moisturizers on dry skin. However, since the
NOA mouse is a mutant strain in which almost no sebum is produced by the sebaceous glands, the oil component may have had a significant effect on the experimental results. Further studies are therefore needed. In the present study, the moisturizing effect was assessed at $24 \mathrm{~h}$ after application. This is because it was not expected that the test article would have an immediate effect and because it was anticipated that the covering effect of the test article might make it difficult to obtain accurate measurements soon after application [24]. The time of measurement should be selected taking into consideration the drug form of the test article and the time of appearance of the expected effect. Mice that were individually housed for 4 weeks from 5-9 weeks of age were used in the present study because of the large variation in the water content of the stratum corneum in mice up to 9 weeks of age under both individual and group housing conditions and also because a relatively stable water content was observed from 9 weeks of age onward in a preliminary experiment.

To date, there has been no suitable animal model for the evaluation of topical moisturizers, and a method using the flexor surface of the forearm of healthy persons has been widely employed [7, 22, 28]. Various in vitro evaluation systems using human stratum corneum sheets [16] and the extracted skin of pigs [12] have also been described. Sato et al. [18] reported that the water content of the stratum corneum of hairless mice was decreased by rearing them under extremely dry conditions (10\% humidity) and that the water content of the stratum corneum was restored by the application of petrolatum. The applicability of this system to the evaluation of topical moisturizers should be considered. However, it is difficult to maintain $10 \%$ environmental humidity in ordinary animal rooms or laboratories, and this system could therefore be employed at only a few institutions. Our evaluation system employing NOA mice with dry skin due to individual housing is suitable for ordinary animal rooms and laboratories. This system can be used easily at any institution, with no need to maintain experimental conditions of temperature and humidity that can be problematic when humans are used and regardless of seasonal factors. Since the NOA mouse is a hairless mutant strain, hair clipping is not required, and animals with dry skin can easily be obtained by rearing them under individual housing conditions. This easily prepared disease model is new 
and significant and can be used as an in vivo evaluation system for performing studies that could previously be conducted only using humans.

The abstract of this report was presented at the 50th Annual Meeting of the Japanese Association for Laboratory Animal Science (2003, Saitama, Japan).

\section{References}

1. Aioi, A., Okuda, M., Matsui, M., Tonogaito, H., and Hamada, K. 2001. Effect of high population density environment on skin barrier function in mice. J. Dermatol. Sci. 25: 189-197.

2. Al'Abadie, M.S., Kent, G.G., and Gawkrodger, D.J. 1994. The relationship between stress and the onset and exacerbation of psoriasis and other skin conditions. $B r . J$. Dermatol. 130: 199-203.

3. Al-Ahmar, H.F. and Kurban, A.K. 1976. Psychological profile of patients with atopic dermatitis. Br.J. Dermatol. 95: 373-377.

4. Allerhand, M.E., Gough, H.G., and Grais, M.L. 1950. Personality factors in neurodermatitis: a preliminary study. Psychosomatic Medicine 12: 386-390.

5. Bartolomucci, A., Palanza, P., Gaspani, L., Limiroli, E., Panerai, A.E., Ceresini, G., Poli, M.D., and Pannigiani, S. 2001. Social status in mice: behavioral, endocrine and immune changes are context dependent. Physiol. Behav. 73: 401-410.

6. Cole, W.C., Roth, H.L., and Sachs, L.B. 1988. Group psychotherapy as an aid in the medical treatment of eczema. J. Am. Acad. Dermatol. 18: 286-291.

7. Gloor, M., Hirsch, G., and Willebrandt, U. 1981. On the use of infrared spectroscopy for the in vivo measurement of the water content of the horny layer after application of dermatologic ointments. Arch. Dermatol. Res. 271: 305313.

8. Hanifin, J.M. 1984. Atopic dermatitis. J. Allergy Clin. Immunol. 73: 211-222.

9. Hariya, T., Hirao, T., Katsuyama, M., Ichikawa, H., Aihara, M., and Ikezawa, Z. 2000. A relationship between a psychosomatic and a skin condition in patients with atopic dermatitis. Jpn. J. Allergol. 49: 463-471 (in Japanese).

10. Higaki, Y., Akikawa, J., Yoshihara, N., Kawamoto, K., Kamo, T., Horikawa, M., and Kawashima, M. 2001. The role of scratching behavior and psychosocial stress in intractable atopic dermatitis. Jpn. J. Dermatol. 111: 837842 (in Japanese).

11. Ishida, H., Mitsui, K., Nukaya, H., Matsumoto, K., and Tsuji, K. 2003. Study of active substances involved in skin dysfunction induced by crowding stress. I. Effect of crowding and isolation on some physiological variables, skin function and skin blood perfusion in hairless mice. Biol. Pharm. Bull. 26: 170-181.

12. Kawano, K., Ohno, H., Asakawa, K., and Deguchi, H. 1993. Evaluation of moisturizing efficacy of urea lotion (UL10): comparison with UREPEARL ${ }^{\circledR}$ and UL-10 lotion base.
The Clinical Report 27: 475-479 (in Japanese).

13. Kondo, T., Shiomoto, Y., Kondo, T., and Kubo, S. 1997. The NOA mouse: a new hair-deficient mutant: a possible animal model of allergic dermatitis. Mouse Genome 95: 698-700.

14. Kondo, T., Kondo, T., Shiomoto, Y., and Momii, A. 2005. Effects of housing conditions on the development of wet skin lesions in the NOA mouse. Exp. Anim. 54: 149-154.

15. McLaughlin, J.T., Shoemaker, R.J., and Guy, W.B. 1953. Personality factors in adult atopic eczema. Arch. Derm. Syph. 68: 506-516.

16. Obata, M. and Tagami, H. 1990. A rapid in vitro test to assess skin moisturizers. J. Soc. Cosmet. Chem. 41: 235241.

17. Obermayer, M.E., Blair, R., Fiske, C., Levitt, N., Rush, S., Storkan, M.A., and Glenn, E. 1952. Correlation of emotional status and reactivity to cutaneous stimuli in functional dermatoses. Arch. Derm. Syph. 65: 291-302.

18. Sato, J., Denda, M., Ashida, Y., and Koyama, J. 1998. Loss of water from the stratum corneum induces epidermal DNA synthesis in hairless mice. Arch. Dermatol. Res. 290: 634-637.

19. Seville, R.S. 1977. Psoriasis and stress. Br. J. Dermatol. 97: 279-302.

20. Seville, R.S. 1978. Psoriasis and stress II. Br. J. Dermatol. 98: 151-153.

21. Tagami, H., Ohi, M., Iwatsuki, K., Kanamara, Y., Yamada, M., and Ichijo, B. 1980. Evaluation of the skin surface hydration in vivo by electrical measurement. J. Invest. Dermatol. 75: 500-507.

22. Tagami, H. 1982. Electrical measurement of the water content of the skin surface: functional analysis of the hygroscopic property and water-holding capacity of the stratum corneum in vivo and technique for assessing moisturizing efficacy. Cosmet. Toiletr. 97: 39-47.

23. Tagami, H., Kanamaru, Y., Inoue, K., Suehisa, S., Inoue, F., lwatsuki, K., Yoshikuni, K., and Yamada, M. 1982. Water sorption-desorption test of the skin in vivo for functional assessment of the stratum corneum. J. Invest. Dermatol. 78: 425-428.

24. Tagami, H. 1994. Quantitative measurements of water concentration of the stratum corneum in vivo by highfrequency current. Acta Derm. Venereol. (Stockh.) 185: 29-33.

25. Tsuchiya, T. and Horii, I. 1995. Immobilization-induced stress decreases lipogenesis in sebaceous glands as well as plasma testosterone level in male Syrian hamsters. Psychoneuroendocrinology 20: 221-230.

26. Uehara, M. and Miyauchi, H. 1984. The morphologic characteristics of dry skin in atopic dermatitis. Arch. Dermatol. 120: 1186-1190.

27. Werner, Y. 1986. The water content of the stratum corneum in patients with atopic dermatitis: measurement with the corneometer CM 420. Acta Derm. Venereol. (Stockh.) 66: 281-284.

28. Wu, M.S., Yee, D.J., and Sullivan, M.E. 1983. Effect of a skin moisturizer on the water distribution in human stratum corneum. J. Invest. Dermatol. 81: 446-448. 